\title{
Data report: carbon and oxygen isotopes of bulk sediments from the off-mound site, Hole U1318B'
}

\author{
Chizuru Takashima, ${ }^{2}$ Akihiro Kano, ${ }^{2}$ and Xianghui $\mathrm{Li}^{3}$
}

\section{Chapter contents}

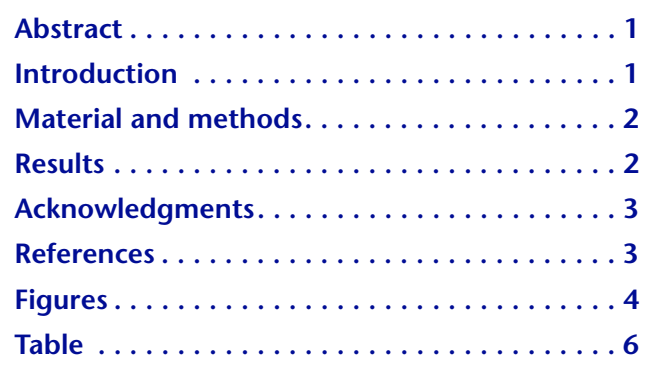

\footnotetext{
${ }^{1}$ Takashima, C., Kano, A., and Li, X., 2009. Data report: carbon and oxygen isotopes of bulk sediments from the off-mound site, Hole U1318B. In Ferdelman, T.G., Kano, A., Williams, T., Henriet, J.-P., and the Expedition 307 Scientists, Proc. IODP, 307: Washington, DC (Integrated Ocean Drilling Program Management International, Inc.). doi:10.2204/iodp.proc.307.202.2009 ${ }^{2}$ Graduate School of Social and Cultural Studies, Kyushu University, Motooka 744, Fukuoka 8190395, Japan. Correspondence author: ctakashima@scs.kyushu-u.ac.jp ${ }^{3}$ Department of Earth Science, State Key Laboratory for Mineral Deposits Research, Nanjing University, 22 Hankou Road, Nanjing, Jiangsu 210093, China.
}

\begin{abstract}
The first and only attempt to drill throughout a deepwater coral mound was carried out during Integrated Ocean Drilling Program Expedition 307. Along with Site U1317 at the summit of Challenger Mound, offshore Ireland, two reference sites were selected in order to construct the regional stratigraphic framework. Sediments from near-shelf Site U1318 are mainly composed of silty clay and sands of Miocene-Pleistocene age. We analyzed oxygen and carbon isotopic composition of the bulk sediments for the upper $90.5 \mathrm{~m}$ in order to understand the source of the carbonate fraction. $\delta^{13} \mathrm{C}$ values range from $-3.15 \%$ to $1.20 \%$ (versus Vienna Peedee belemnite [VPDB]) and $\delta^{18} \mathrm{O}$ values range from $-4.59 \%$ o to $1.87 \%$ o (versus VPDB). A distinct stratigraphic trend is that the Miocene sediments record higher $\delta^{18} \mathrm{O}$ values. In general, the carbonate fraction of Site U1318 sediments have much lower oxygen and higher carbon isotopic values than the mound sediments, and the oxygen values are similar to those of Cretaceous chalk.
\end{abstract}

\section{Introduction}

Recent oceanographic exploration in the northeast Atlantic has discovered widespread and diverse coral ecosystems in deepwater environments of the several provinces. The Belgica mound province in Porcupine Seabight is one of them and has 47 exposed mounds (De Mol et al., 2007), of which Challenger Mound (Site U1317) was drilled during Integrated Ocean Drilling Program (IODP) Expedition 307 in May 2005. In order to link the mound sediments to the regional stratigraphic framework, two additional sites were selected: Site U1316 at the foot of the mound and Site U1318 upslope from the mound.

Miocene-Pleistocene sediments were recovered from three holes at Site U1318 $\left(51^{\circ} 26.16^{\prime} \mathrm{N}, 11^{\circ} 33.0^{\prime} \mathrm{W} ; 423 \mathrm{~m}\right.$ water depth) and were divided into three lithologic units at two distinct unconformable horizons. In the deepest hole (U1318B), the uppermost lithologic Unit 1, consisting of $74.5 \mathrm{~m}$ thick silty clay, was divided into three subunits (1A, 1B, and $1 \mathrm{C})$ based on relative dominance of laminated/bioturbated textures. The underlying $8 \mathrm{~m}$ thick Unit 2 consists of medium-fine sand interbedded with silty clay layers and contains a $10-20 \mathrm{~cm}$ thick oyster bed. The lowermost Unit 3 is composed of $155 \mathrm{~m}$ thick siltstone that was divided into three 
subunits (3A, 3B, and 3C) (see the "Site U1318" chapter).

The age of these units were first evaluated shipboard using calcareous nannofossil biostratigraphy, which assigned Units 1 and 2 to the late middle Pleistocene-late Pleistocene Emiliana huxleyi Zone $(<0.26$ $\mathrm{Ma}$ ) and the early Pleistocene (small Gephyrocapsa Zone), respectively. ${ }^{87} \mathrm{Sr} /{ }^{86} \mathrm{Sr}$ ratios of the molluscan skeletons evaluated the age of Unit 2 (early Pleistocene) and Unit 3 (Pliocene-middle Miocene) (Kano et al., 2007). The age model of Unit 3 was later refined by dinoflagellate cyst biostratigraphy (Louwye et al., 2008), indicating that the top of the unit is late Serravallian $(\sim 12.0 \mathrm{Ma})$.

Because Site U1318 is upslope from Challenger Mound, it involves sedimentological information on the shelf slope where deepwater coral mounds are developed. We focused on the carbonate fraction, for which potential sources are calcareous nannofossils, benthic foraminifers, planktonic foraminifers, and Cretaceous chalk from ice-rafted transportation. These sources have different carbon and oxygen isotopic values. We report carbon and oxygen isotopic compositions of the bulk sediments from Hole $\mathrm{U} 1318 \mathrm{~B}$ in order to provide some information on the sediment sources to the upslope site of Challenger Mound.

\section{Material and methods}

The analyzed interval of Hole U1318B ranges from 0.05 meters below seafloor (mbsf) (interval 307U1318B-1H-1, $5 \mathrm{~cm}$; top of Unit 1) to $90.5 \mathrm{mbsf}$ (interval 307-U1318B-10H-CC, $23 \mathrm{~cm}$; Subunit 3A). The sampling interval was $20 \mathrm{~cm}$. After excluding large skeletal grains, 422 samples were dried and powdered for carbon and oxygen isotope measurement.

Isotopic compositions were measured using mass spectrometry (Finnigan Mat Delta Plus) coupled with an automatic gas separation system (Gas Bench). In this system, the samples were reacted with purified $\mathrm{H}_{3} \mathrm{PO}_{4}$ under $1 \mathrm{~atm}$ He in a sealed glass bottle. Reaction temperature and time were $55^{\circ} \mathrm{C}$ and $3 \mathrm{~h}$, respectively. The reactant $\mathrm{CO}_{2}$ was, together with $\mathrm{He}$, sent to the gas chromatograph, which separated components such as $\mathrm{H}_{2} \mathrm{O}$. The separated $\mathrm{CO}_{2}$ was introduced into the mass spectrometer, where its isotopic compositions were measured. The measured carbon and oxygen isotopic ratios were normalized with the laboratory standard (Solnhofen Limestone; $\left.\delta^{13} \mathrm{C}=-0.47 \% 0, \delta^{18} \mathrm{O}=-5.04 \% 0\right)$ that was calibrated against National Bureau of Standards (NBS) $19\left(\delta^{13} \mathrm{C}\right.$ $=+1.95 \%$ o, $\delta^{18} \mathrm{O}=-2.20 \%$ versus Vienna Peedee belemnite). Repeated measurements of the laboratory standard $(n=22)$ showed that the reproducibility of $\delta^{13} \mathrm{C}$ and $\delta^{18} \mathrm{O}$ was within \pm 0.20 and $\pm 0.27 \%$, respectively. Outliers were measured twice to check their accuracy.

\section{Results}

Raw data and stratigraphic profiles of carbon and oxygen isotopes are shown in Table T1 and Figure F1.

$\delta^{13} \mathrm{C}$ values range from $-3.2 \%$ to $1.2 \%$, which is generally higher and more stable than the $\delta^{13} \mathrm{C}$ range of the mound section $(-5.3 \%$ o to $-1.5 \%$ ) (Kano et al., 2007). The values fluctuate from $-1.0 \%$ to $1.0 \%$ in Subunits $1 \mathrm{~A}$ and $1 \mathrm{~B}$ and record the lowest value (around $-3 \%$ ) in Subunit 1C. From the lower part of Subunit $1 \mathrm{C}$ to Subunit $3 \mathrm{~A}, \delta^{13} \mathrm{C}$ values increase gradually.

$\delta^{18} \mathrm{O}$ values range from $-4.6 \%$ to $1.9 \%$ (Table $\mathrm{T} 1$; Fig. F1). The profile shows a slight increasing trend from the top of Subunit 1A to Unit 2, with three peak horizons in Subunit 1A. At the Unit 2/Subunit $3 \mathrm{~A}$ boundary, $\delta^{18} \mathrm{O}$ values represent an abrupt increase from around $-2.5 \%$ to $+1.5 \%$.

Cross-plots of carbon and oxygen isotopes (Fig. F2) represent differences in the values among the stratigraphic units. The most distinct pattern is the distribution of the Subunit 3A samples, which is much higher in $\delta^{18} \mathrm{O}$ and slightly higher in $\delta^{13} \mathrm{C}$ than other units. Most Unit 1 samples and many Unit 2 samples were plotted in a small domain of $-2.0 \%$ o to $-4.5 \%$ o in $\delta^{18} \mathrm{O}$ and $0.8 \%$ to $-1.0 \%$ in $\delta^{13} \mathrm{C}$.

Figure $\mathbf{F} 2$ also shows ranges in $\delta^{18} \mathrm{O}$ and $\delta^{13} \mathrm{C}$ values of potential carbonate sources, including Pleistocene benthic foraminifers in the North Atlantic $\left(\delta^{18} \mathrm{O}=\right.$ $2.5 \%$ o to $5.5 \%$; $\delta^{13} \mathrm{C}=-0.5 \%$ o to $-1.5 \%$ ) (Venz et al., $1999)$, planktonic foraminifers from Site U1317 $\left(\delta^{18} \mathrm{O}\right.$ $=-0.8 \%$ to $1.4 \%$; $\delta^{13} \mathrm{C}=0.5 \%$ o to $-1.5 \%$ ) (Sakai et al., 2009), and Cretaceous chalk in Southeast England $\left(\delta^{18} \mathrm{O}=-1.8 \%\right.$ o to approximately $-3.5 \%$; $\delta^{13} \mathrm{C}=$ $1.3 \%$ to $\sim 3.0 \%$ ) (Jenkyns et al., 1994). It also shows the results of bulk sediments from on-mound Site U1317 (Takashima et al., 2006).

Variation in $\delta^{18} \mathrm{O}$ and $\delta^{13} \mathrm{C}$ values in our plots can be explained by variation in relative importance of source carbonates. The Miocene (Subunit 3A) plots indicated that much of the carbonate was derived from foraminifers, whereas the values (especially $\delta^{18} \mathrm{O}$ ) of Subunits 1 and 2 are similar to the values of Cretaceous chalk (Fig. F2). This trend is consistent with the common occurrence of reworked Cretaceous nannofossils (see the "Site U1318" chapter). The $2 \%$ difference in $\delta^{13} \mathrm{C}$ between the bulk Site U1318 sediments and southeast England chalk (Fig. 
F2) suggests that there might be substantial sources with lower $\delta^{13} \mathrm{C}$ values that cannot be specified in this study. The three $\delta^{18} \mathrm{O}$ peak horizons in Subunit $1 \mathrm{~A}$ may indicate the low- $\delta^{18} \mathrm{O}$ fraction of an eroded chalk. Sediments in Units 1 and 2 contain an abundance of terrestrial particles that are associated with ice-rafted debris (see the "Site U1318" chapter). Therefore, the changes in oxygen and carbon isotopic values from Unit 3 to Units 1 and 2 likely reflect intensified glaciation during the late Pliocene and early Pleistocene.

\section{Acknowledgments}

We thank Professor Dierk Hebbeln for providing constructive comments to the manuscript. Thanks are expressed to the Integrated Ocean Drilling Program (IODP) for samples and data and the Center for Deep Earth Exploration (CDEX) for funding efforts. We would like to thank the members of scientific party and the crew of the JOIDES Resolution during IODP Expedition 307. Japan Agency for MarineEarth Science and Technology supported this study.

\section{References}

De Mol, B., Kozachenko, M., Wheeler, A., Alvares, H., Henriet, J.-P., and Olu-Le Roy, K., 2005. Thérèse Mound: a case study of coral bank development in the Belgica mound province, Porcupine Seabight. Int. J. Earth Sci., 96(1):103-120. doi:10.1007/s00531-005-0496-X

Jenkyns, H.C., Gale, A.S., and Corfield, R.M., 1994. Carbon- and oxygen-isotope stratigraphy of the English Chalk and Italian Scaglia and its palaeoclimatic signifi- cance. Geol. Mag., 131(1):1-34. doi:10.1017/ S0016756800010451

Kano, A., Ferdelman, T.G., Williams, T., Henriet, J.-P., Ishikawa, T., Kawagoe, N., Takashima, C., Kakizaki, Y., Abe, K., Sakai, S., Browning, E.L., Li, X., and the Integrated Ocean Drilling Program Expedition 307 Scientists, 2007. Age constraints on the origin and growth history of a deep-water coral mound in the northeast Atlantic drilled during Integrated Ocean Drilling Program Expedition 307. Geology, 35(11):1051-1054. doi:10.1130/G23917A.1

Louwye, S., Foubert, A., Mertens, K., Van Rooij, D., and the IODP Expedition 307 Scientific Party, 2008. Integrated stratigraphy and palaeoecology of the lower and middle Miocene of the Porcupine Basin. Geol. Mag., 145(3):321344. doi:10.1017/S0016756807004244

Sakai, S., Kano, A., and Abe, K., 2009. Origin, glacial-interglacial responses, and controlling factors of a cold-water coral mound in NE Atlantic. Paleoceanography, 24(2):PA2213. doi:10.1029/2008PA001695

Takashima, C., Li, X., Kawai, T., Kano, A., and IODP Expedition 307 Scientists, 2006. Oxygen and carbon isotopic profile of a deep-water coral mound section drilled in IODP Expedition 307 [17th International Sedimentological Congress, Fukuoka, Japan, 27 August-1 September 2006].

Venz, K.A., Hodell, D.A., Stanton, C., and Warnke, D.A., 1999. A 1.0 Myr record of glacial North Atlantic intermediate water variability from ODP Site 982 in the Northeast Atlantic. Paleoceanography, 14(1):42-52. doi:10.1029/1998PA900013

Initial receipt: 7 June 2008

Acceptance: 27 May 2009

Publication: 15 July 2009

MS 307-202 
Figure F1. Stratigraphic profiles of carbon and oxygen isotopic values, Hole U1318B.

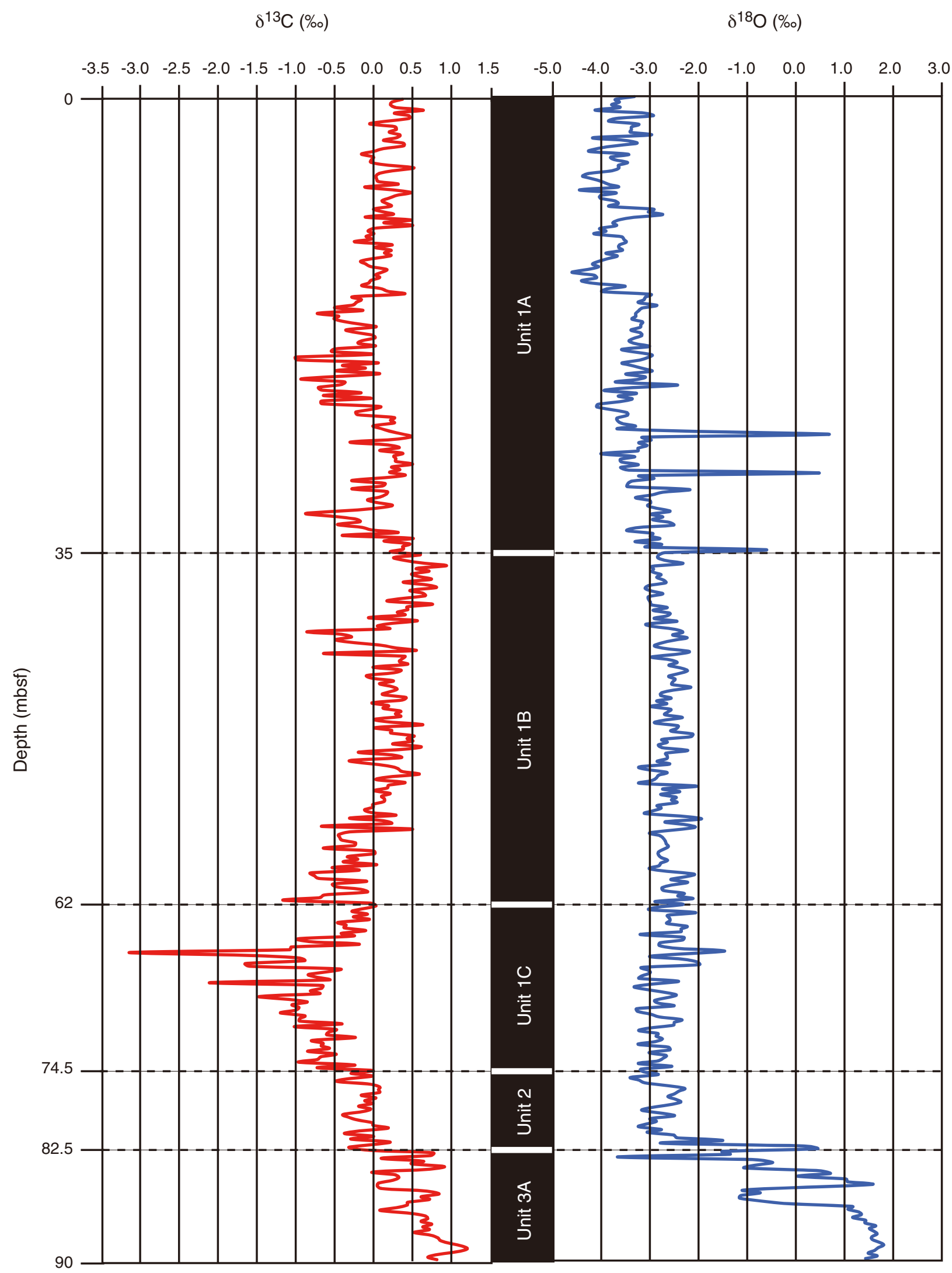


Figure F2. Cross-plot of carbon and oxygen values, Hole U1318B. For comparison, the isotopic range of potential sources (A-C) and bulk mound sediments from Hole U1317E (D) (Takashima et al., 2006) are also shown. A = benthic foraminifer Cibicidoides sp. from ODP Site 982, B = planktonic foraminifer Globigerina bulloides from Hole U1317E (Sakai et al., 2009), C = Cretaceous chalk from southeast England (Jenkyns et al., 1994).

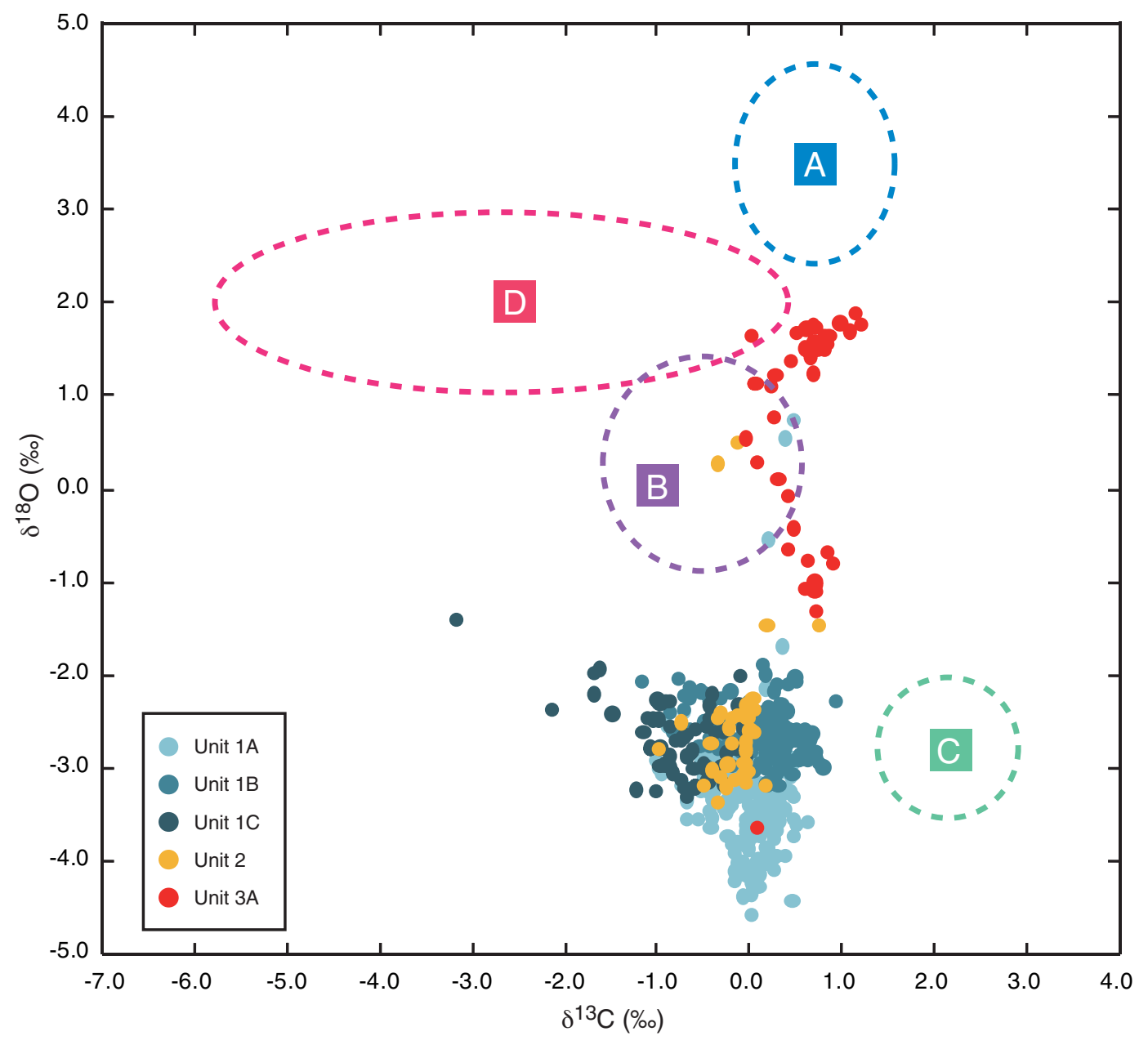


Table T1. Carbon and oxygen isotopic values, Hole U1318B. (Continued on next two pages.)

\begin{tabular}{|c|c|c|}
\hline $\begin{array}{l}\text { Core, section, } \\
\text { interval }(\mathrm{cm})\end{array}$ & $\begin{array}{l}\delta^{13} \mathrm{C} \\
(\% 0)\end{array}$ & $\begin{array}{l}\delta^{18} \mathrm{O} \\
(\% 0)\end{array}$ \\
\hline \multicolumn{3}{|l|}{ 307-U1318B- } \\
\hline $1 \mathrm{H}-1,5-6$ & 0.37 & -3.28 \\
\hline $1 \mathrm{H}-1,25-26$ & 0.23 & -3.68 \\
\hline $1 \mathrm{H}-1,45-46$ & 0.21 & -3.60 \\
\hline $1 \mathrm{H}-1,65-66$ & 0.27 & -3.77 \\
\hline $1 \mathrm{H}-1,85-86$ & 0.63 & -3.59 \\
\hline $1 \mathrm{H}-1,105-106$ & 0.26 & -4.10 \\
\hline $1 \mathrm{H}-1,125-126$ & 0.45 & -3.09 \\
\hline $1 \mathrm{H}-1,145-146$ & 0.45 & -2.90 \\
\hline $1 \mathrm{H}-2,10-11$ & 0.16 & -3.69 \\
\hline $1 \mathrm{H}-2,25-26$ & -0.06 & -3.82 \\
\hline $1 \mathrm{H}-2,45-46$ & 0.26 & -3.21 \\
\hline $1 \mathrm{H}-2,65-66$ & 0.28 & -3.35 \\
\hline $1 \mathrm{H}-2,85-86$ & 0.19 & -3.35 \\
\hline $1 \mathrm{H}-2,105-106$ & 0.33 & -3.37 \\
\hline $1 \mathrm{H}-2,125-126$ & 0.29 & -2.95 \\
\hline $1 \mathrm{H}-2,145-146$ & 0.12 & -4.15 \\
\hline $1 \mathrm{H}-3,10-11$ & 0.37 & -3.46 \\
\hline $1 \mathrm{H}-3,25-26$ & 0.38 & -3.24 \\
\hline $1 \mathrm{H}-3,45-46$ & 0.12 & -3.74 \\
\hline $1 \mathrm{H}-3,65-66$ & -0.01 & -4.07 \\
\hline $1 \mathrm{H}-3,85-86$ & -0.17 & -4.22 \\
\hline $1 \mathrm{H}-4,15-16$ & -0.01 & -3.41 \\
\hline $1 \mathrm{H}-4,35-36$ & -0.04 & -3.78 \\
\hline $1 \mathrm{H}-4,75-76$ & -0.05 & -3.70 \\
\hline $2 \mathrm{H}-1,15-16$ & 0.16 & -3.43 \\
\hline $2 \mathrm{H}-1,35-36$ & 0.51 & -3.62 \\
\hline $2 \mathrm{H}-1,55-56$ & 0.26 & -3.61 \\
\hline $2 \mathrm{H}-1,75-76$ & 0.06 & -3.75 \\
\hline $2 \mathrm{H}-1,85-86$ & 0.02 & -4.24 \\
\hline $2 \mathrm{H}-1,105-106$ & 0.03 & -4.37 \\
\hline $2 \mathrm{H}-1,125-126$ & 0.05 & -4.14 \\
\hline $2 \mathrm{H}-1,145-146$ & 0.31 & -3.96 \\
\hline $2 \mathrm{H}-2,5-6$ & -0.12 & -3.82 \\
\hline $2 \mathrm{H}-2,25-26$ & 0.22 & -3.64 \\
\hline $2 \mathrm{H}-2,45-46$ & 0.47 & -4.43 \\
\hline $2 \mathrm{H}-2,65-66$ & 0.29 & -3.68 \\
\hline $2 \mathrm{H}-2,85-86$ & 0.20 & -4.00 \\
\hline $2 \mathrm{H}-2,105-106$ & 0.10 & -4.00 \\
\hline $2 \mathrm{H}-2,125-126$ & 0.16 & -3.69 \\
\hline $2 \mathrm{H}-2,145-146$ & 0.21 & -3.63 \\
\hline $2 \mathrm{H}-3,15-16$ & -0.01 & -3.81 \\
\hline $2 \mathrm{H}-3,35-36$ & 0.11 & -2.89 \\
\hline $2 \mathrm{H}-3,55-56$ & 0.24 & -3.00 \\
\hline $2 \mathrm{H}-3,75-76$ & -0.12 & -2.71 \\
\hline $2 \mathrm{H}-3,95-96$ & 0.48 & -3.33 \\
\hline $2 \mathrm{H}-3,115-116$ & 0.12 & -3.64 \\
\hline $2 \mathrm{H}-3,135-136$ & 0.49 & -3.74 \\
\hline $2 \mathrm{H}-4,5-6$ & 0.01 & -3.67 \\
\hline $2 \mathrm{H}-4,25-26$ & -0.08 & -4.01 \\
\hline $2 \mathrm{H}-4,45-46$ & -0.01 & -3.88 \\
\hline $2 \mathrm{H}-4,55-56$ & -0.11 & -4.13 \\
\hline $2 \mathrm{H}-4,65-66$ & -0.04 & -3.56 \\
\hline $2 \mathrm{H}-4,85-86$ & -0.25 & -3.51 \\
\hline $2 \mathrm{H}-4,105-106$ & 0.23 & -3.46 \\
\hline $2 \mathrm{H}-4,125-126$ & 0.01 & -3.60 \\
\hline $2 \mathrm{H}-4,145-146$ & 0.22 & -3.62 \\
\hline $2 \mathrm{H}-5,10-11$ & 0.13 & -3.54 \\
\hline $2 \mathrm{H}-5,30-31$ & 0.21 & -3.88 \\
\hline $2 \mathrm{H}-5,50-51$ & -0.00 & -3.64 \\
\hline $2 \mathrm{H}-5,70-71$ & -0.17 & -3.84 \\
\hline $2 \mathrm{H}-5,90-91$ & -0.11 & -4.03 \\
\hline $2 \mathrm{H}-5,110-111$ & -0.05 & -4.16 \\
\hline $2 \mathrm{H}-5,130-131$ & 0.16 & -4.04 \\
\hline $2 \mathrm{H}-6,0-1$ & 0.10 & -4.28 \\
\hline $2 \mathrm{H}-6,20-21$ & 0.02 & -4.59 \\
\hline $2 \mathrm{H}-6,40-41$ & 0.07 & -4.13 \\
\hline $2 \mathrm{H}-6,60-61$ & -0.04 & -4.08 \\
\hline $2 \mathrm{H}-6,80-81$ & -0.07 & -4.39 \\
\hline
\end{tabular}

\begin{tabular}{|c|c|c|}
\hline $\begin{array}{l}\text { Core, section, } \\
\text { interval }(\mathrm{cm})\end{array}$ & $\begin{array}{l}\delta^{13} \mathrm{C} \\
(\% 0)\end{array}$ & $\begin{array}{c}\delta^{18} \mathrm{O} \\
(\% 0)\end{array}$ \\
\hline $2 \mathrm{H}-6,100-101$ & -0.16 & -4.13 \\
\hline $2 \mathrm{H}-6,120-121$ & 0.09 & -3.48 \\
\hline $2 \mathrm{H}-6,140-141$ & 0.17 & -3.86 \\
\hline 2H-CC, 10-11 & 0.38 & -3.96 \\
\hline $3 \mathrm{H}-1,55-56$ & -0.28 & -2.95 \\
\hline $3 \mathrm{H}-1,75-76$ & -0.17 & -3.06 \\
\hline $3 \mathrm{H}-1,95-96$ & -0.23 & -3.10 \\
\hline $3 \mathrm{H}-1,115-116$ & -0.27 & -3.21 \\
\hline $3 \mathrm{H}-1,135-136$ & -0.51 & -2.82 \\
\hline $3 \mathrm{H}-2,10-11$ & -0.15 & -3.14 \\
\hline $3 \mathrm{H}-2,30-31$ & -0.73 & -3.20 \\
\hline $3 \mathrm{H}-2,50-51$ & -0.46 & -3.27 \\
\hline $3 \mathrm{H}-2,70-71$ & -0.52 & -3.26 \\
\hline $3 \mathrm{H}-2,90-91$ & -0.40 & -3.34 \\
\hline $3 \mathrm{H}-2,110-111$ & -0.18 & -3.12 \\
\hline $3 \mathrm{H}-2,130-131$ & 0.02 & -3.16 \\
\hline $3 \mathrm{H}-2,149-150$ & -0.36 & -3.17 \\
\hline $3 \mathrm{H}-3,20-21$ & -0.24 & -3.36 \\
\hline $3 \mathrm{H}-3,40-41$ & -0.02 & -3.16 \\
\hline $3 \mathrm{H}-3,60-61$ & 0.01 & -3.14 \\
\hline $3 \mathrm{H}-3,80-81$ & -0.16 & -3.30 \\
\hline $3 \mathrm{H}-3,100-101$ & -0.21 & -3.40 \\
\hline $3 \mathrm{H}-3,120-121$ & 0.01 & -3.25 \\
\hline $3 \mathrm{H}-3,140-141$ & -0.51 & -2.98 \\
\hline $3 \mathrm{H}-4,10-11$ & -0.55 & -3.55 \\
\hline $3 \mathrm{H}-4,30-31$ & -0.02 & -3.27 \\
\hline $3 \mathrm{H}-4,50-51$ & -1.01 & -2.93 \\
\hline $3 \mathrm{H}-4,70-71$ & -0.99 & -3.02 \\
\hline $3 \mathrm{H}-4,90-91$ & 0.04 & -3.20 \\
\hline $3 \mathrm{H}-4,110-111$ & -0.41 & -3.55 \\
\hline $3 \mathrm{H}-4,130-131$ & -0.11 & -3.31 \\
\hline $3 \mathrm{H}-4,148-149$ & -0.50 & -3.11 \\
\hline $3 \mathrm{H}-5,20-21$ & 0.07 & -2.93 \\
\hline $3 \mathrm{H}-5,40-41$ & -0.44 & -3.47 \\
\hline $3 \mathrm{H}-5,60-61$ & -0.95 & -3.07 \\
\hline $3 \mathrm{H}-5,80-81$ & -0.38 & -3.24 \\
\hline $3 \mathrm{H}-5,100-101$ & -0.42 & -3.66 \\
\hline $3 \mathrm{H}-5,120-121$ & -0.72 & -2.40 \\
\hline $3 \mathrm{H}-5,140-141$ & -0.68 & -3.37 \\
\hline $3 \mathrm{H}-6,10-11$ & -0.17 & -3.92 \\
\hline $3 \mathrm{H}-6,30-31$ & -0.66 & -3.25 \\
\hline $3 \mathrm{H}-6,50-51$ & -0.04 & -3.63 \\
\hline $3 \mathrm{H}-6,70-71$ & -0.68 & -3.34 \\
\hline $3 \mathrm{H}-6,90-91$ & -0.68 & -3.57 \\
\hline $3 \mathrm{H}-6,110-111$ & 0.07 & -4.04 \\
\hline $3 \mathrm{H}-6,130-131$ & -0.03 & -4.07 \\
\hline $3 \mathrm{H}-6,148-149$ & -0.24 & -3.75 \\
\hline $3 \mathrm{H}-7,20-21$ & -0.22 & -3.45 \\
\hline $3 \mathrm{H}-7,40-41$ & 0.26 & -3.43 \\
\hline $3 \mathrm{H}-\mathrm{CC}, 10-11$ & 0.21 & -3.65 \\
\hline $4 \mathrm{H}-1,60-61$ & 0.26 & -3.64 \\
\hline $4 \mathrm{H}-1,80-81$ & -0.01 & -3.55 \\
\hline $4 \mathrm{H}-1,100-101$ & 0.09 & -3.27 \\
\hline $4 \mathrm{H}-1,120-121$ & 0.20 & -3.62 \\
\hline $4 \mathrm{H}-1,140-141$ & 0.36 & -1.70 \\
\hline $4 \mathrm{H}-2,10-11$ & 0.48 & 0.73 \\
\hline $4 \mathrm{H}-2,30-31$ & 0.25 & -3.12 \\
\hline $4 \mathrm{H}-2,50-51$ & -0.31 & -2.94 \\
\hline $4 \mathrm{H}-2,70-71$ & 0.18 & -3.20 \\
\hline $4 \mathrm{H}-2,90-91$ & 0.32 & -3.03 \\
\hline $4 \mathrm{H}-2,110-111$ & 0.07 & -3.22 \\
\hline $4 \mathrm{H}-2,130-131$ & 0.36 & -3.22 \\
\hline $4 \mathrm{H}-2,149-150$ & 0.25 & -3.99 \\
\hline $4 \mathrm{H}-3,20-21$ & 0.28 & -3.29 \\
\hline $4 \mathrm{H}-3,40-41$ & 0.28 & -3.58 \\
\hline $4 \mathrm{H}-3,60-61$ & 0.49 & -3.56 \\
\hline $4 \mathrm{H}-3,80-81$ & 0.22 & -3.21 \\
\hline $4 \mathrm{H}-3,100-101$ & 0.33 & -3.58 \\
\hline $4 \mathrm{H}-3,120-121$ & 0.20 & -3.41 \\
\hline
\end{tabular}

\begin{tabular}{|c|c|c|}
\hline $\begin{array}{l}\text { Core, section, } \\
\text { interval }(\mathrm{cm})\end{array}$ & $\begin{array}{l}\delta^{13} \mathrm{C} \\
(\% \circ)\end{array}$ & $\begin{array}{l}\delta^{18} \mathrm{O} \\
(\%)\end{array}$ \\
\hline $4 \mathrm{H}-3,140-141$ & 0.40 & 0.54 \\
\hline $4 \mathrm{H}-4,10-11$ & 0.10 & -3.18 \\
\hline $4 \mathrm{H}-4,30-31$ & -0.29 & -2.89 \\
\hline $4 \mathrm{H}-4,50-51$ & 0.13 & -3.35 \\
\hline $4 \mathrm{H}-4,70-71$ & 0.10 & -3.43 \\
\hline $4 \mathrm{H}-4,90-91$ & -0.29 & -3.44 \\
\hline $4 \mathrm{H}-4,110-111$ & 0.17 & -2.16 \\
\hline $4 \mathrm{H}-4,130-131$ & 0.15 & -2.70 \\
\hline $4 \mathrm{H}-4,145-146$ & 0.07 & -2.93 \\
\hline $4 \mathrm{H}-5,15-16$ & -0.09 & -3.27 \\
\hline $4 \mathrm{H}-5,35-36$ & 0.04 & -2.97 \\
\hline $4 \mathrm{H}-5,55-56$ & 0.23 & -2.96 \\
\hline $4 \mathrm{H}-5,75-76$ & 0.05 & -3.02 \\
\hline $4 \mathrm{H}-5,95-96$ & -0.37 & -2.79 \\
\hline $4 \mathrm{H}-5,115-116$ & -0.89 & -2.55 \\
\hline $4 \mathrm{H}-5,135-136$ & -0.53 & -2.94 \\
\hline $4 \mathrm{H}-6,5-6$ & -0.24 & -2.70 \\
\hline $4 \mathrm{H}-6,27-28$ & -0.18 & -2.92 \\
\hline $4 \mathrm{H}-6,45-46$ & -0.48 & -2.56 \\
\hline $4 \mathrm{H}-6,65-66$ & -0.17 & -2.48 \\
\hline $4 \mathrm{H}-6,85-86$ & -0.03 & -3.29 \\
\hline $4 \mathrm{H}-6,105-106$ & 0.30 & -3.45 \\
\hline $4 \mathrm{H}-6,127-129$ & -0.41 & -2.91 \\
\hline $4 \mathrm{H}-6,145-146$ & 0.49 & -2.96 \\
\hline $4 \mathrm{H}-\mathrm{CC}, 20-21$ & 0.12 & -2.77 \\
\hline $5 \mathrm{H}-1,74-75$ & 0.45 & -3.28 \\
\hline $5 \mathrm{H}-1,95-96$ & 0.36 & -2.73 \\
\hline $5 \mathrm{H}-1,115-116$ & 0.37 & -3.04 \\
\hline $5 \mathrm{H}-1,135-136$ & 0.21 & -0.55 \\
\hline $5 \mathrm{H}-2,10-11$ & 0.59 & -2.66 \\
\hline $5 \mathrm{H}-2,30-31$ & 0.25 & -2.80 \\
\hline $5 \mathrm{H}-2,50-51$ & 0.38 & -2.80 \\
\hline $5 \mathrm{H}-2,70-71$ & 0.60 & -2.60 \\
\hline $5 \mathrm{H}-2,90-91$ & 0.93 & -2.29 \\
\hline $5 \mathrm{H}-2,110-111$ & 0.54 & -2.93 \\
\hline $5 \mathrm{H}-2,130-131$ & 0.71 & -2.89 \\
\hline $5 \mathrm{H}-2,149-150$ & 0.48 & -2.92 \\
\hline $5 \mathrm{H}-3,20-21$ & 0.58 & -2.74 \\
\hline $5 \mathrm{H}-3,40-41$ & 0.73 & -2.83 \\
\hline $5 \mathrm{H}-3,60-61$ & 0.37 & -2.68 \\
\hline $5 \mathrm{H}-3,80-81$ & 0.67 & -2.64 \\
\hline $5 \mathrm{H}-3,100-101$ & 0.80 & -3.00 \\
\hline $5 \mathrm{H}-3,120-121$ & 0.46 & -3.07 \\
\hline $5 \mathrm{H}-3,140-141$ & 0.62 & -2.93 \\
\hline $5 \mathrm{H}-4,10-11$ & 0.65 & -2.70 \\
\hline $5 \mathrm{H}-4,30-31$ & 0.34 & -3.00 \\
\hline $5 \mathrm{H}-4,50-51$ & 0.18 & -3.00 \\
\hline $5 \mathrm{H}-4,70-71$ & 0.75 & -2.96 \\
\hline $5 \mathrm{H}-4,90-91$ & 0.42 & -2.94 \\
\hline $5 \mathrm{H}-4,110-111$ & 0.43 & -2.61 \\
\hline $5 \mathrm{H}-4,130-131$ & 0.30 & -2.90 \\
\hline $5 \mathrm{H}-4,149-150$ & 0.39 & -2.56 \\
\hline $5 \mathrm{H}-5,20-21$ & -0.08 & -2.61 \\
\hline $5 \mathrm{H}-5,40-41$ & 0.55 & -2.88 \\
\hline $5 \mathrm{H}-5,60-61$ & 0.23 & -2.41 \\
\hline $5 \mathrm{H}-5,80-81$ & 0.04 & -3.04 \\
\hline $5 \mathrm{H}-5,100-101$ & 0.19 & -2.85 \\
\hline $5 \mathrm{H}-5,120-121$ & -0.85 & -2.42 \\
\hline $5 \mathrm{H}-5,140-141$ & -0.42 & -2.29 \\
\hline $5 \mathrm{H}-6,10-11$ & -0.29 & -2.50 \\
\hline $5 \mathrm{H}-6,30-31$ & -0.50 & -2.20 \\
\hline $5 \mathrm{H}-6,50-51$ & -0.28 & -2.52 \\
\hline $5 \mathrm{H}-6,70-71$ & 0.09 & -2.75 \\
\hline $5 \mathrm{H}-6,90-91$ & 0.28 & -2.88 \\
\hline $5 \mathrm{H}-7,10-11$ & 0.51 & -2.61 \\
\hline $5 \mathrm{H}-7,30-31$ & -0.66 & -2.15 \\
\hline $5 \mathrm{H}-7,50-51$ & 0.39 & -2.42 \\
\hline $5 \mathrm{H}-7,70-71$ & 0.36 & -2.94 \\
\hline $5 \mathrm{H}-\mathrm{CC}, 10-11$ & 0.32 & -2.55 \\
\hline
\end{tabular}


Table T1 (continued). (Continued on next page.)

\begin{tabular}{|c|c|c|}
\hline $\begin{array}{l}\text { Core, section, } \\
\text { interval }(\mathrm{cm})\end{array}$ & $\begin{array}{l}\delta^{13} \mathrm{C} \\
(\% 0)\end{array}$ & $\begin{array}{l}\delta^{18} \mathrm{O} \\
(\% 0)\end{array}$ \\
\hline $5 \mathrm{H}-\mathrm{CC}, 30-31$ & 0.42 & -2.40 \\
\hline $6 \mathrm{H}-1,10-11$ & -0.02 & -2.58 \\
\hline $6 \mathrm{H}-1,30-31$ & 0.34 & -2.33 \\
\hline $6 \mathrm{H}-1,50-51$ & 0.26 & -2.19 \\
\hline $6 \mathrm{H}-1,70-71$ & -0.10 & -2.36 \\
\hline $6 \mathrm{H}-1,90-91$ & -0.03 & -2.58 \\
\hline $6 \mathrm{H}-1,110-111$ & 0.25 & -2.45 \\
\hline $6 \mathrm{H}-1,130-131$ & 0.07 & -2.49 \\
\hline $6 \mathrm{H}-1,149-150$ & 0.22 & -2.51 \\
\hline $6 \mathrm{H}-2,15-16$ & 0.29 & -2.12 \\
\hline $6 \mathrm{H}-2,35-36$ & 0.20 & -2.46 \\
\hline $6 \mathrm{H}-2,55-56$ & 0.11 & -2.73 \\
\hline $6 \mathrm{H}-2,75-76$ & 0.41 & -2.75 \\
\hline $6 \mathrm{H}-2,95-96$ & 0.36 & -2.53 \\
\hline $6 \mathrm{H}-2,115-116$ & -0.03 & -2.83 \\
\hline $6 \mathrm{H}-2,135-136$ & 0.18 & -2.62 \\
\hline $6 \mathrm{H}-3,5-6$ & 0.11 & -2.95 \\
\hline $6 \mathrm{H}-3,25-26$ & 0.34 & -2.54 \\
\hline $6 \mathrm{H}-3,45-46$ & 0.27 & -2.60 \\
\hline $6 \mathrm{H}-3,65-66$ & 0.34 & -2.66 \\
\hline $6 \mathrm{H}-3,85-86$ & 0.00 & -2.30 \\
\hline $6 \mathrm{H}-3,105-106$ & 0.20 & -2.68 \\
\hline $6 \mathrm{H}-3,125-126$ & 0.62 & -2.87 \\
\hline $6 \mathrm{H}-3,145-146$ & 0.01 & -2.39 \\
\hline $6 \mathrm{H}-4,15-16$ & 0.22 & -2.41 \\
\hline $6 \mathrm{H}-4,35-36$ & 0.21 & -2.53 \\
\hline $6 \mathrm{H}-4,55-56$ & 0.51 & -2.09 \\
\hline $6 \mathrm{H}-4,75-76$ & 0.42 & -2.12 \\
\hline $6 \mathrm{H}-4,95-96$ & 0.49 & -2.72 \\
\hline $6 \mathrm{H}-4,115-116$ & 0.23 & -2.61 \\
\hline $6 \mathrm{H}-4,135-136$ & 0.61 & -2.80 \\
\hline $6 \mathrm{H}-5,5-6$ & 0.35 & -2.72 \\
\hline $6 \mathrm{H}-5,25-26$ & -0.21 & -2.18 \\
\hline $6 \mathrm{H}-5,45-46$ & 0.24 & -2.64 \\
\hline $6 \mathrm{H}-5,65-66$ & 0.34 & -2.61 \\
\hline $6 \mathrm{H}-5,85-86$ & -0.31 & -2.62 \\
\hline $6 \mathrm{H}-5,105-106$ & -0.11 & -2.82 \\
\hline $6 \mathrm{H}-5,125-126$ & 0.19 & -2.56 \\
\hline $6 \mathrm{H}-5,145-146$ & 0.31 & -3.19 \\
\hline $6 \mathrm{H}-6,15-16$ & 0.35 & -3.01 \\
\hline $6 \mathrm{H}-6,35-36$ & 0.58 & -2.62 \\
\hline $6 \mathrm{H}-6,55-56$ & 0.18 & -2.79 \\
\hline $6 \mathrm{H}-\mathrm{CC}, 15-16$ & 0.02 & -2.83 \\
\hline $7 \mathrm{H}-1,75-76$ & 0.40 & -2.93 \\
\hline 7H-1, 95-96 & 0.18 & -3.18 \\
\hline $7 \mathrm{H}-1,115-116$ & 0.17 & -2.01 \\
\hline 7H-1, 135-136 & 0.00 & -2.71 \\
\hline $7 \mathrm{H}-2,5-6$ & 0.20 & -2.35 \\
\hline $7 \mathrm{H}-2,25-26$ & 0.09 & -2.75 \\
\hline $7 \mathrm{H}-2,45-46$ & 0.13 & -2.43 \\
\hline $7 \mathrm{H}-2,65-66$ & 0.12 & -2.53 \\
\hline $7 \mathrm{H}-2,85-86$ & -0.02 & -2.41 \\
\hline 7H-2, 105-106 & -0.01 & -2.82 \\
\hline 7H-2, 125-126 & -0.13 & -2.74 \\
\hline 7H-2, 145-146 & -0.03 & -2.91 \\
\hline 7H-3, 15-16 & 0.28 & -3.07 \\
\hline $7 \mathrm{H}-3,35-36$ & -0.32 & -2.26 \\
\hline 7H-3, 55-56 & 0.13 & -1.91 \\
\hline $7 \mathrm{H}-3,75-76$ & 0.22 & -2.66 \\
\hline $7 \mathrm{H}-3,95-96$ & -0.68 & -2.24 \\
\hline $7 \mathrm{H}-3,115-116$ & 0.49 & -2.04 \\
\hline 7H-3, 135-136 & -0.30 & -2.49 \\
\hline $7 \mathrm{H}-4,5-6$ & -0.46 & -2.98 \\
\hline $7 \mathrm{H}-4,25-26$ & -0.44 & -2.76 \\
\hline $7 \mathrm{H}-4,45-46$ & -0.42 & -2.69 \\
\hline 7H-4, 65-66 & -0.24 & -2.65 \\
\hline $7 \mathrm{H}-4,85-86$ & -0.26 & -2.63 \\
\hline 7H-4, 105-106 & -0.65 & -2.59 \\
\hline $7 \mathrm{H}-4,125-126$ & -0.01 & -2.69 \\
\hline
\end{tabular}

\begin{tabular}{|c|c|c|}
\hline $\begin{array}{l}\text { Core, section, } \\
\text { interval }(\mathrm{cm})\end{array}$ & $\begin{array}{l}\delta^{13} \mathrm{C} \\
(\%)\end{array}$ & $\begin{array}{c}\delta^{18} \mathrm{O} \\
(\% 0)\end{array}$ \\
\hline 7H-4, 145-146 & 0.00 & -2.80 \\
\hline 7H-5, 15-16 & -0.34 & -2.80 \\
\hline 7H-5, 35-36 & -0.21 & -2.74 \\
\hline 7H-5, 55-56 & -0.40 & -2.60 \\
\hline $7 \mathrm{H}-5,75-76$ & 0.03 & -2.74 \\
\hline 7H-5, 95-96 & -0.54 & -2.78 \\
\hline 7H-5, 115-116 & -0.20 & -2.99 \\
\hline 7H-5, 135-136 & -0.82 & -2.62 \\
\hline $7 \mathrm{H}-6,5-6$ & -0.77 & -2.06 \\
\hline $7 \mathrm{H}-6,25-26$ & -0.71 & -2.24 \\
\hline $7 \mathrm{H}-6,45-46$ & -0.10 & -2.54 \\
\hline 7H-6, 65-66 & -0.53 & -2.19 \\
\hline 7H-6, 85-86 & -0.50 & -2.52 \\
\hline 7H-6, 105-106 & -0.16 & -2.72 \\
\hline 7H-6, 125-126 & -0.10 & -2.69 \\
\hline 7H-6, 145-146 & -0.64 & -2.26 \\
\hline 7H-CC, 15-16 & -0.70 & -2.39 \\
\hline $7 \mathrm{H}-\mathrm{CC}, 35-36$ & -1.16 & -2.09 \\
\hline $8 \mathrm{H}-1,15-16$ & -0.04 & -2.87 \\
\hline $8 \mathrm{H}-1,35-36$ & 0.02 & -2.28 \\
\hline $8 \mathrm{H}-1,55-56$ & -0.14 & -2.78 \\
\hline $8 \mathrm{H}-1,75-76$ & -0.29 & -2.98 \\
\hline $8 \mathrm{H}-1,95-96$ & -0.09 & -2.03 \\
\hline $8 \mathrm{H}-1,115-116$ & -0.27 & -2.61 \\
\hline $8 \mathrm{H}-1,135-136$ & -0.06 & -2.56 \\
\hline $8 \mathrm{H}-2,5-6$ & -0.46 & -2.55 \\
\hline $8 \mathrm{H}-2,25-26$ & -0.36 & -2.62 \\
\hline $8 \mathrm{H}-2,45-46$ & -0.39 & -2.21 \\
\hline $8 \mathrm{H}-2,65-66$ & -0.11 & -2.33 \\
\hline $8 \mathrm{H}-2,85-86$ & -0.43 & -2.33 \\
\hline $8 \mathrm{H}-2,105-106$ & -0.27 & -3.17 \\
\hline $8 \mathrm{H}-2,125-126$ & -0.99 & -2.27 \\
\hline $8 \mathrm{H}-2,145-146$ & -0.86 & -2.29 \\
\hline $8 \mathrm{H}-3,15-16$ & -0.19 & -2.76 \\
\hline $8 \mathrm{H}-3,35-36$ & -1.07 & -2.77 \\
\hline $8 \mathrm{H}-3,55-56$ & -1.09 & -2.46 \\
\hline $8 \mathrm{H}-3,75-76$ & -3.15 & -1.42 \\
\hline $8 \mathrm{H}-3,95-96$ & -1.68 & -2.21 \\
\hline $8 \mathrm{H}-3,115-116$ & -0.96 & -2.98 \\
\hline $8 \mathrm{H}-3,135-136$ & -0.90 & -2.28 \\
\hline $8 \mathrm{H}-4,5-6$ & -1.66 & -1.98 \\
\hline $8 \mathrm{H}-4,25-26$ & -1.61 & -1.94 \\
\hline $8 \mathrm{H}-4,75-76$ & -0.44 & -3.14 \\
\hline $8 \mathrm{H}-4,95-96$ & -0.60 & -3.00 \\
\hline $8 \mathrm{H}-4,115-116$ & -0.85 & -2.97 \\
\hline $8 \mathrm{H}-4,135-136$ & -0.74 & -3.13 \\
\hline $8 \mathrm{H}-5,15-16$ & -0.59 & -3.19 \\
\hline $8 \mathrm{H}-5,35-36$ & -2.13 & -2.38 \\
\hline $8 \mathrm{H}-5,55-56$ & -0.67 & -2.89 \\
\hline $8 \mathrm{H}-5,75-76$ & -0.68 & -3.30 \\
\hline $8 \mathrm{H}-5,95-96$ & -0.82 & -3.05 \\
\hline $8 \mathrm{H}-5,115-116$ & -0.71 & -2.68 \\
\hline $8 \mathrm{H}-5,135-136$ & -1.48 & -2.42 \\
\hline $8 \mathrm{H}-6,5-6$ & -1.14 & -2.62 \\
\hline $8 \mathrm{H}-6,25-26$ & -0.86 & -2.87 \\
\hline $8 \mathrm{H}-6,45-46$ & -1.06 & -2.79 \\
\hline $8 H-6,65-66$ & -0.97 & -2.48 \\
\hline $8 \mathrm{H}-6,85-86$ & -1.01 & -3.25 \\
\hline $8 \mathrm{H}-6,105-106$ & -1.21 & -3.23 \\
\hline $8 \mathrm{H}-6,125-126$ & -0.90 & -2.96 \\
\hline $8 \mathrm{H}-\mathrm{CC}, 10-11$ & -0.96 & -2.77 \\
\hline $8 \mathrm{H}-\mathrm{CC}, 30-31$ & -0.96 & -2.31 \\
\hline $9 \mathrm{H}-1,10-12$ & -0.42 & -2.47 \\
\hline $9 \mathrm{H}-1,30-32$ & -1.03 & -2.48 \\
\hline $9 \mathrm{H}-1,50-52$ & -0.50 & -2.96 \\
\hline $9 \mathrm{H}-1,70-72$ & -0.59 & -3.20 \\
\hline $9 \mathrm{H}-1,90-92$ & -0.61 & -2.80 \\
\hline $9 \mathrm{H}-1,110-112$ & -0.25 & -2.84 \\
\hline $9 \mathrm{H}-1,130-132$ & -0.80 & -2.71 \\
\hline
\end{tabular}

\begin{tabular}{|c|c|c|}
\hline $\begin{array}{l}\text { Core, section, } \\
\text { interval }(\mathrm{cm})\end{array}$ & $\begin{array}{l}\delta^{13} \mathrm{C} \\
(\% 0)\end{array}$ & $\begin{array}{c}\delta^{18} \mathrm{O} \\
(\% 0)\end{array}$ \\
\hline $9 \mathrm{H}-1,148-150$ & -0.66 & -2.86 \\
\hline $9 \mathrm{H}-2,40-42$ & -0.68 & -3.22 \\
\hline $9 \mathrm{H}-2,60-62$ & -0.58 & -2.59 \\
\hline $9 \mathrm{H}-2,80-82$ & -0.86 & -2.56 \\
\hline $9 \mathrm{H}-2,120-122$ & -0.49 & -2.99 \\
\hline $9 \mathrm{H}-2,140-142$ & -0.69 & -2.64 \\
\hline $9 \mathrm{H}-3,12-14$ & -0.73 & -2.70 \\
\hline $9 \mathrm{H}-3,32-34$ & -0.97 & -2.80 \\
\hline $9 \mathrm{H}-3,52-54$ & -0.25 & -3.21 \\
\hline $9 \mathrm{H}-3,70-72$ & -0.74 & -2.51 \\
\hline $9 \mathrm{H}-3,90-92$ & -0.02 & -3.16 \\
\hline $9 \mathrm{H}-3,100-102$ & -0.30 & -3.10 \\
\hline $9 \mathrm{H}-3,110-112$ & -0.03 & -2.80 \\
\hline $9 \mathrm{H}-3,130-132$ & -0.33 & -3.37 \\
\hline $9 \mathrm{H}-3,148-150$ & -0.48 & -3.19 \\
\hline $9 \mathrm{H}-4,20-22$ & -0.04 & -3.08 \\
\hline $9 \mathrm{H}-4,40-42$ & 0.07 & -2.61 \\
\hline $9 \mathrm{H}-4,55-57$ & 0.05 & -2.25 \\
\hline $9 \mathrm{H}-4,75-77$ & 0.07 & -2.37 \\
\hline $9 \mathrm{H}-4,95-97$ & -0.17 & -2.46 \\
\hline $9 \mathrm{H}-4,110-112$ & 0.02 & -2.60 \\
\hline $9 \mathrm{H}-4,130-132$ & -0.12 & -2.43 \\
\hline $9 \mathrm{H}-4,150-152$ & -0.02 & -2.33 \\
\hline $9 \mathrm{H}-5,20-22$ & -0.20 & -2.58 \\
\hline $9 \mathrm{H}-5,40-42$ & -0.05 & -2.95 \\
\hline $9 \mathrm{H}-5,60-62$ & -0.16 & -3.14 \\
\hline $9 \mathrm{H}-5,80-82$ & -0.40 & -2.74 \\
\hline $9 \mathrm{H}-5,100-102$ & -0.33 & -2.46 \\
\hline $9 \mathrm{H}-6,20-22$ & -0.22 & -2.96 \\
\hline $9 \mathrm{H}-6,40-42$ & -0.02 & -2.83 \\
\hline $9 \mathrm{H}-\mathrm{CC}, 5-7$ & -0.01 & -3.04 \\
\hline $9 \mathrm{H}-\mathrm{CC}, 25-27$ & 0.18 & -3.20 \\
\hline $9 \mathrm{H}-\mathrm{CC}, 45-47$ & -0.18 & -2.73 \\
\hline $10 \mathrm{H}-1,5-7$ & -0.38 & -3.03 \\
\hline $10 \mathrm{H}-1,25-27$ & -0.01 & -2.47 \\
\hline $10 \mathrm{H}-1,45-47$ & -0.30 & -2.41 \\
\hline $10 \mathrm{H}-1,65-67$ & 0.20 & -1.46 \\
\hline $10 \mathrm{H}-1,85-87$ & -0.02 & -2.72 \\
\hline $10 \mathrm{H}-1,105-107$ & -0.33 & 0.28 \\
\hline $10 \mathrm{H}-1,125-127$ & -0.11 & 0.50 \\
\hline $10 \mathrm{H}-1,145-147$ & 0.76 & -1.47 \\
\hline $10 \mathrm{H}-2,5-7$ & 0.73 & -1.32 \\
\hline $10 \mathrm{H}-2,20-22$ & 0.09 & -3.64 \\
\hline $10 \mathrm{H}-2,40-42$ & 0.63 & -0.78 \\
\hline $10 \mathrm{H}-2,55-57$ & 0.48 & -0.43 \\
\hline $10 \mathrm{H}-2,70-72$ & 0.90 & -0.81 \\
\hline $10 \mathrm{H}-2,85-87$ & 0.71 & -1.01 \\
\hline $10 \mathrm{H}-2,100-102$ & -0.02 & 0.54 \\
\hline $10 \mathrm{H}-2,120-122$ & 0.27 & 0.77 \\
\hline $10 \mathrm{H}-2,145-147$ & 0.32 & 0.09 \\
\hline $10 \mathrm{H}-3,15-17$ & 0.24 & 1.09 \\
\hline $10 \mathrm{H}-3,35-37$ & 0.05 & 1.12 \\
\hline $10 \mathrm{H}-3,55-57$ & 0.04 & 1.63 \\
\hline $10 \mathrm{H}-3,75-77$ & 0.09 & 0.28 \\
\hline $10 \mathrm{H}-3,95-97$ & 0.71 & -1.03 \\
\hline $10 \mathrm{H}-3,115-117$ & 0.83 & -0.68 \\
\hline $10 \mathrm{H}-3,135-137$ & 0.60 & -1.08 \\
\hline $10 \mathrm{H}-4,5-7$ & 0.71 & -1.11 \\
\hline $10 \mathrm{H}-4,25-27$ & 0.43 & -0.66 \\
\hline $10 \mathrm{H}-4,45-47$ & 0.43 & -0.09 \\
\hline $10 \mathrm{H}-4,115-117$ & 0.28 & 1.22 \\
\hline $10 \mathrm{H}-4,135-137$ & 0.07 & 1.13 \\
\hline $10 \mathrm{H}-4,148-150$ & 0.45 & 1.36 \\
\hline $10 \mathrm{H}-5,18-20$ & 0.66 & 1.41 \\
\hline $10 \mathrm{H}-5,35-37$ & 0.69 & 1.23 \\
\hline $10 \mathrm{H}-5,55-57$ & 0.60 & 1.50 \\
\hline $10 \mathrm{H}-5,75-77$ & 0.74 & 1.48 \\
\hline $10 \mathrm{H}-5,95-97$ & 0.62 & 1.71 \\
\hline $10 \mathrm{H}-5,105-107$ & 0.71 & 1.58 \\
\hline
\end{tabular}


Table T1 (continued).

\begin{tabular}{lll}
\hline $\begin{array}{l}\text { Core, section, } \\
\text { interval }(\mathrm{cm})\end{array}$ & $\begin{array}{l}\delta^{13} \mathrm{C} \\
(\% 0)\end{array}$ & $\begin{array}{l}\delta^{18} \mathrm{O} \\
(\% \circ)\end{array}$ \\
\hline $10 \mathrm{H}-5,120-122$ & 0.51 & 1.67 \\
$10 \mathrm{H}-5,135-137$ & 0.72 & 1.74 \\
$10 \mathrm{H}-5,148-150$ & 0.82 & 1.63 \\
$10 \mathrm{H}-6,20-22$ & 0.85 & 1.63 \\
$10 \mathrm{H}-6,40-42$ & 0.98 & 1.78 \\
$10 \mathrm{H}-6,60-62$ & 1.13 & 1.87 \\
$10 \mathrm{H}-6,80-82$ & 1.20 & 1.76 \\
$10 \mathrm{H}-6,100-102$ & 1.07 & 1.68 \\
$10 \mathrm{H}-6,120-122$ & 0.83 & 1.55 \\
$10 \mathrm{H}-\mathrm{CC}, 5-7$ & 0.69 & 1.74 \\
$10 \mathrm{H}-\mathrm{CC}, 23-25$ & 0.81 & 1.49 \\
\hline
\end{tabular}

